\title{
Iridium-Catalyzed Silylation of Five-Membered Heteroarenes: High Sterically Derived Selectivity from a Pyridyl-Imidazoline Ligand
}

\author{
Caleb Karmel ${ }^{\ddagger}$, Camille Z. Rubel ${ }^{\ddagger}$, Elena V. Kharitonova ${ }^{\star}$, and John F. Hartwig* \\ Department of Chemistry, University of California, Berkeley, California, 94720, United States \\ Supporting Information Placeholder
}

\begin{abstract}
The steric effects of substituents on five-membered rings are less pronounced than those on six-membered rings because of the difference in bond angles. Thus, the regioselectivities of reactions that occur with selectivities dictated by steric effects, such as the borylation of $\mathrm{C}-\mathrm{H}$ bonds, have been poor in many cases. We report that the silylation of five-membered ring heteroarenes occurs with high sterically derived regioselectivity when catalyzed by the combination of $[\operatorname{Ir}(\operatorname{cod})(\mathrm{OMe})]_{2}$ and a phenanthroline ligand or a new pyridylimidazoline ligand that further increases the regioselectivity. The silylation reactions with these catalysts produce high yields of heteroarylsilanes from functionalization at the most sterically accessible $\mathrm{C}-\mathrm{H}$ bonds of these rings under conditions that the borylation of $\mathrm{C}-\mathrm{H}$ bonds with previously reported catalysts formed mixtures of products or products that are unstable. The heteroarylsilane products undergo cross-coupling reactions and substitution reactions with ipso selectivity to generate heteroarenes that bear halogen, aryl and perfluoroalkyl substituents.
\end{abstract}

The application of $\mathrm{C}-\mathrm{H}$ bond functionalization to synthetic chemistry requires the selective activation of a single $\mathrm{C}-\mathrm{H}$ bond among multiple $\mathrm{C}-\mathrm{H}$ bonds that possess different steric and electronic properties.1,2 $\mathrm{A}$ common strategy for controlling the selectivity of catalytic reactions that functionalize the $\mathrm{C}-\mathrm{H}$ bonds of arenes is to incorporate a functional group on the arene that coordinates to a transition-metal catalyst and directs the reaction to $\mathrm{C}-\mathrm{H}$ bonds that are ortho, meta or para to that functional group. ${ }^{3}$

Undirected functionalizations of the $\mathrm{C}-\mathrm{H}$ bonds of arenes occur with selectivities that are determined by the steric and electronic properties of the various types of $\mathrm{C}-\mathrm{H}$ bonds. ${ }^{4}$ The most classical undirected functionalizations include uncatalyzed or Lewis-acid catalyzed electrophilic aromatic substitution (EAS) processes. In general, these reactions occur at the most electron-rich $\mathrm{C}-\mathrm{H}$ bonds, and the steric properties of the groups impart a secondary influence on selectivity. ${ }^{5}$

\section{Figure 1}

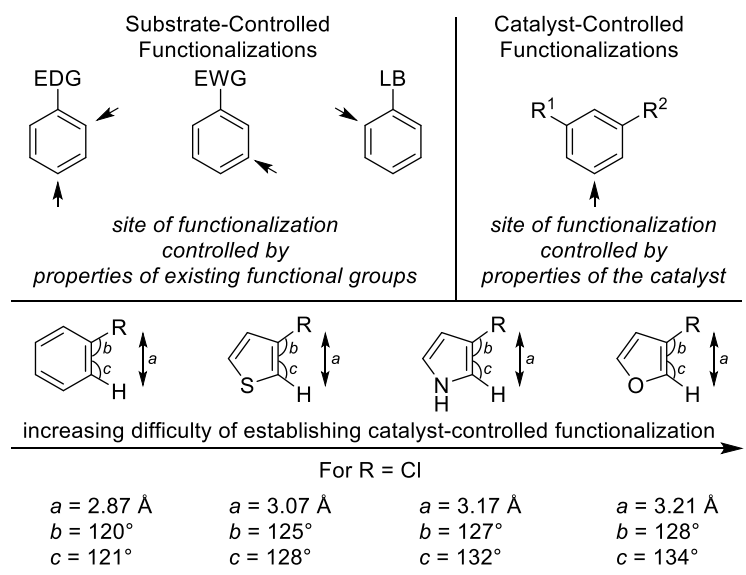

In contrast, the silylations and borylations of the $\mathrm{C}-\mathrm{H}$ bonds of arenes catalyzed by iridium complexes of bipyridine or phenanthroline ligands generally occur at the most sterically accessible and acidic $\mathrm{C}-\mathrm{H}$ bonds. ${ }^{6}$ The regioselectivity of these reactions is almost exclusively determined by the steric properties of the arene; these catalysts selectively functionalize $\mathrm{C}-\mathrm{H}$ bonds that are distal to functional groups in preference to $\mathrm{C}-\mathrm{H}$ bonds that are more acidic, but proximal to functional groups. Thus, symmetrical 1,2-disubstituted or unsymmetrical 1,3-disubstituted or 1,2,3-trisubstituted arenes undergo borylation at the $\mathrm{C}-\mathrm{H}$ meta to the nearest substituent (Figure 1).

The regioselectivity of the functionalization of heteroarenes is more complex, and the influence of steric properties on the selectivity of the functionalization of 5 -membered heteroarenes is smaller, than that of 6membered rings because the more acute bond angles in five-membered rings cause the distances between the substituents to be longer than the distances between substituents in 6-membered rings (Figure 1). Thus, catalytic functionalization of a single $\mathrm{C}-\mathrm{H}$ bond in a five-membered ring heteroarene with high regioselectivity derived from steric effects is rare due to the distinct electronic properties of the positions of 
heteroarenes and the weaker steric influence in fivemembered ring structures.

Consistent with this trend, the selectivity of iridium-catalyzed borylation reactions of five-membered ring heteroarenes is poor. The current borylations of five-membered heteroarenes, in many cases, form isomers from the functionalization of sites ortho to small or medium-sized substitutents. ${ }^{7}$ Even the recently developed catalyst of Smith and Maleczka that contains a novel $\mathrm{N}-\mathrm{N}$ ligand produced mixtures of products from the borylations of 3-substituted thiophenes, including products from diborylation, even if the thiophene is in excess. ${ }^{8}$

The identification of reactions that functionalize $\mathrm{C}$ $\mathrm{H}$ bonds in five-membered-ring heteroarenes regioselectively is important because these structural motifs are common in pharmaceuticals, electronic materials, agrochemicals, and natural products. ${ }^{9}$ The regioselective silylation and borylation of heteroarenes would be particularly useful because silylarenes and aryl boronic esters undergo hydroxylation, ${ }^{10}$ etherification, ${ }^{11}$ amination, ${ }^{12}$ cyanation, ${ }^{13}$ halogenation, ${ }^{14}$ and arylation, ${ }^{15}$ and analogous derivatization of heteroarenes would provide an array of heteroarenes with substitution patterns that are derived from the catalytic system. The silylation of heteroarenes can be particularly valuable because the heteroarylsilanes are often more stable than the corresponding heteroarylboronates. ${ }^{16}$

We report silylations of five-membered heteroarenes catalyzed by an iridium complex that contains a pyridyl-imidazoline ligand, and also silylationscatalyzed by a recently disclosed complex of 2,9$\mathrm{Me}_{2}$-phenanthroline ( $\mathrm{Me}_{2}$ phen). We demonstrate that reactions with these catalysts produce high yields of products that are formed from functionalization of the most sterically accessible $\mathrm{C}-\mathrm{H}$ bonds of five-membered heteroarenes under conditions in which borylation reactions produce mixtures of products. We also illustrate the versatility of the heteroarylsilanes by developing conditions for ipso substitution reactions to produce the corresponding five-membered-ring heteroarenes bearing halogen, aryl and perfluoroalkyl substituents.

\section{Reaction Development}

To achieve the undirected functionalization of fivemembered heteroarenes with regioselectivity that is dictated by steric effects, we investigated reactions catalyzed by iridium complexes of a series of ligands that contain nitrogen donors. Two of the ligands, $\mathbf{L} \mathbf{1}$ and $\mathbf{L 2}$, are based on pyridyl oxazoline structures, and two of the ligands, $\mathbf{L} \mathbf{3}$ and $\mathbf{L} \mathbf{4}$, are based on pyridyl imidazoline structures. Ligand $\mathbf{L} \mathbf{4}$ is new, and none of the ligands have been reported for the borylation or silylation of aromatic $\mathrm{C}-\mathrm{H}$ bonds. We included pyridyl imidazoline structures in our study because the nitrogen atoms should be more electron donating than those in the pyridyl oxazoline structures, and substituents on the nitrogen in the imidazoline could influence the structures of these ligands.

To create a benchmark for our studies on the silylation of five-membered ring heteroarenes, we first measured the yield and selectivity of the borylation of 3-chlorothiophene that was catalyzed by the previously reported combination of $[\operatorname{Ir}(\mathrm{COD})(\mathrm{OMe})]_{2}$ and 4,4'-di-tert-butylbipyridine (dtbpy). ${ }^{17}$ Under standard conditions, the products from functionalization at positions both proximal and distal to the chlorine substituent formed, and the major product resulted from diborylation (Table 1). Similarly, the silylation of 3chlorothiophene under the conditions recently reported by our group with 2,9-dimethylphenanthroline as ligand formed a mixture of products from silylation at both the 2 and 5-positions.

\section{Table 1. Effect of Ligand on Selectivity ${ }^{a}$}

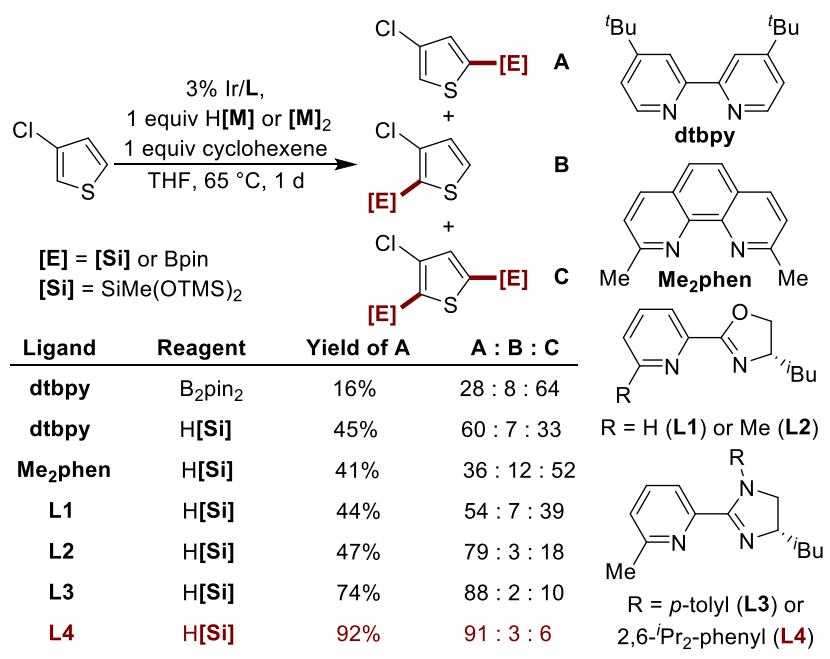

${ }^{a}$ See SI for detailed procedures

In contrast, the silylation of 3-chlorothiophene that was catalyzed by the combination of $[\operatorname{Ir}(\mathrm{COD})(\mathrm{OMe})]_{2}$ and $\mathbf{L} 2$ was selective for functionalization at the 5-position of the heteroarene. This regioselectivity was even higher when the reaction was catalyzed by $[\operatorname{Ir}(\mathrm{COD})(\mathrm{OMe})]_{2}$ and the imidazoline ligand L3 and was the highest when catalyzed by $[\operatorname{Ir}(\mathrm{COD})(\mathrm{OMe})]_{2}$ and imidazoline ligand $\mathbf{L 4}$, which contains a 2,6-iPr-phenyl group on the imidazoline nitrogen. The silylation of 3-chlorothiophene, when catalyzed by iridium and $\mathbf{L 3}$, formed the product from functionalization at the 5-position in 74\% yield, and the silylation of this thiophene, when catalyzed by iridium and $\mathbf{L 4}$, formed the product from functionalization at the 5-position in $90 \%$ yield with a 9:1 
selectivity for this isomer over others and the product from di-functionalization.

We note that this regioselectivity for silylation and chemoselectivity for monosilylation is obtained with limiting heteroarene. The regioselectivity is similar to the that reported for the borylation of the same substrate, but our silylation reaction occurs with the thiophene as limiting reagent. The results on borylation were obtained with an excess of thiophene, and high selectivity for mono-borylation of such a thiophene with the heteroarene as limiting reagent has not been reported with any catalyst. ${ }^{8}$

\section{Reaction Scope}

Having identified a ligand that greatly increases the sensitivity of the iridium-catalyzed silylation of thiophenes to the steric environment of the $\mathrm{C}-\mathrm{H}$ bonds, we studied the selectivity of this catalyst for the silylation of thiophenes that contain varied functional groups. We conducted silylation reactions with the catalyst that contains L4, silylations with the catalyst that contains $\mathrm{Me}_{2} \mathrm{Phen}$, and borylations with the catalyst that contains dtbpy (Table 2). 5-Silyl-thiophenes that contain methyl, methoxy, phenyl, ester, boryl, bromide and iodide substituents in the 3-position were obtained with perfect selectivity for the monosilylation product, with perfect regioselectivity, and in yields ranging from $44 \%$ to $100 \%$ from reactions catalyzed by the combination of iridium and $\mathbf{L 4}$. The ethyl ester of $\mathbf{5}$ and the nitrile of $\mathbf{6}$ were tolerated; no products from reduction of these functional groups were observed. Thiophene $\mathbf{6}$, which contains a small cyano substituent, was converted to the corresponding silylarene with 85:12:3 selectivity for functionalization at the 5-position of 3-cyanothiophene to functionalization at the 2-position to di-silylation. Likewise, heteroarylsilanes that contain halogen substituents were obtained from the corresponding arenes (79) in moderate (3-iodo) to high (3-bromo and 3chloro) yield and without proto-dehalogenation. The reactions of the larger of the three halides gave a single product; the reactions of the 3-chloro isomer gave 94:1 regioselectivity and 95:5 selectivity of monosilylation to disilylation.

Results from the silylations catalyzed by the complex of $\mathrm{Me}_{2}$ Phen and the borylations catalyzed by the complex of dtbpy in Table 2 show the value of the silylation of the catalyst that contains $\mathbf{L 4}$ for obtaining high regioselectivity. The silylation of thiophenes that contain smaller substituents occurred with low selectivity for functionalization at the 5-position $(\mathbf{1}, \mathbf{6}, \mathbf{7})$ when catalyzed by the combination of iridium and $\mathrm{Me}_{2}$ phen. The borylations of these heteroarenes with iridium and dtbpy also produced mixtures of products. Silylations of thiophenes that contain larger phenyl, boryl, ester, bromide, and iodide substituents $(3-5,8,9)$ and that were catalyzed by iridium and $\mathrm{Me}_{2}$ phen occurred with complete selectivity for functionalization at the 5-position of the heteroarene, but the yields were much lower than those catalyzed by iridium and L4. Likewise, borylation with dtbpy was highly selective for the 5-position of 3-phenylthiophene and ethyl 3-thiophenecarboxylate. However, the borylation of all the other thiophenes in Table 2 occurred with low selectivity for the 5-position. These results demonstrate that the catalyst formed from the combination of iridium and $\mathbf{L 4}$ is more active and more selective for the functionalization of the least sterically hindered position of thiophenes than previously reported catalysts.

\section{Table 2. C-H Silylation of Thiophenes ${ }^{a}$}

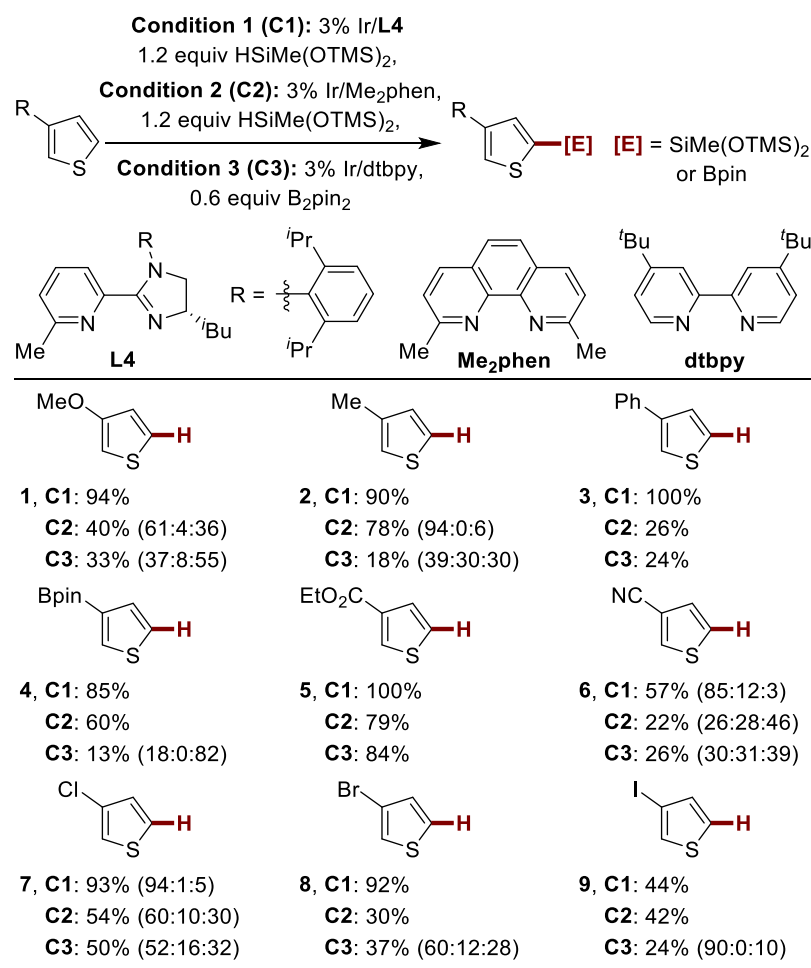

${ }^{a}$ See SI for detailed procedures. Yields correspond to ${ }^{1} \mathrm{H}$ NMR yield of desired product (desired product : undesired, mono- : undesired, di-)

The analogous silylation and borylation reactions of furans and pyrroles are shown in Table 3. The silylations of 3-substituted furans and pyrroles catalyzed by iridium and imidazoline ligand $\mathbf{L} 4$ occurred with perfect selectivity and in good yield (11-15, 60\% to $100 \%$ yield). The silylation of 3-bromofuran (10) occurred with greater than $80 \%$ selectivity for the 5position. In contrast, the silylation or borylation of 3bromofuran under previously reported conditions led to the formation of large amounts of products from functionalization at the 2-position. In addition, the silylations of furans or pyrroles that contain esters at the 3-positions produced significant amounts of products from functionalization at the 2-position when the catalyst contained $\mathrm{Me}_{2}$ phen. The borylations of these 
nitrogen or oxygen-containing heterocycles occurred with universally poor selectivity for the least sterically hindered $\mathrm{C}-\mathrm{H}$ bonds.

\section{Table 3. C-H Silylation of Furans and Pyrroles ${ }^{a}$}

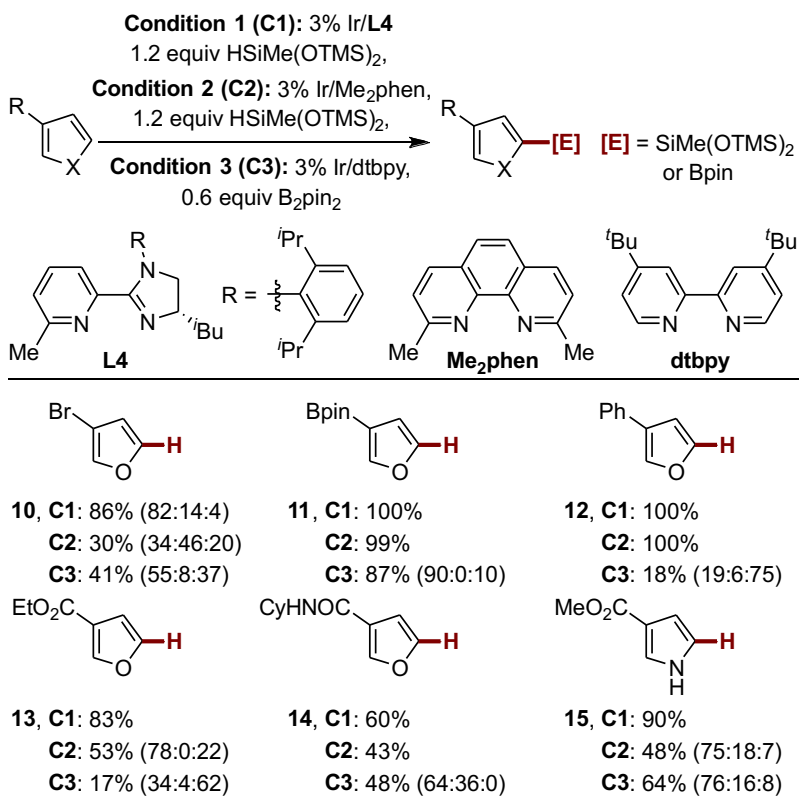

${ }^{a}$ See SI for detailed procedures. Yields correspond to ${ }^{1} \mathrm{H}$ NMR yield of desired product (desired product : undesired, mono- : undesired, di-)

Five-membered ring heterocycles that contain multiple heteroatoms also underwent silylation in good yield and with high selectivity (Table 4). In these cases, the reactions occurred in the highest yields when catalyzed by $[\operatorname{Ir}(\mathrm{COD})(\mathrm{OMe})]_{2}$ and $\mathrm{Me}_{2}$ phen. The functionalized product formed with high regioselectivity from steric effects with electronic effects influencing selectivity when potentially reactive $\mathrm{C}-\mathrm{H}$ bonds were present in similar steric environments.

Three examples of the silylation of pyrazoles are provided in Table 4. The reactions of 1,3-dimethylpyrazole 16, in which the two aromatic $\mathrm{C}-\mathrm{H}$ bonds are in similar steric environments was completely selective for the 5-position, and this selectivity was consistent with the high preference for functionalization $\alpha$ to heteroatoms observed for the functionalization of thiophenes, furans and pyrroles. However, the silylation of pyrazoles that contain larger tert-butyl carbonate or tosyl substituents on nitrogen occurred distal to the nitrogen that bears that substituent. These results indicate that the silylation of azoles occurs with a selectivity that is determined primarily by the steric environment of the $\mathrm{C}-\mathrm{H}$ bonds, with electronic effects imparting a secondary influence.

The silylations of the thiazoles occurred at the position that is most sterically accessible. One might expect that the reaction would occur at the position more distal to the basic nitrogen because borylations of pyridines were shown previously not to form products from reaction at the position alpha to a basic nitrogen atom. ${ }^{18}$ However, the silylation reaction formed a stable product from reaction at the 2-position of 4-substituted thiazoles due to the steric congestion at the 5-position, even if the substituent at the 4-position was as small as a methyl group.

\section{Table 4. C-H Silylation of Azoles ${ }^{a}$}

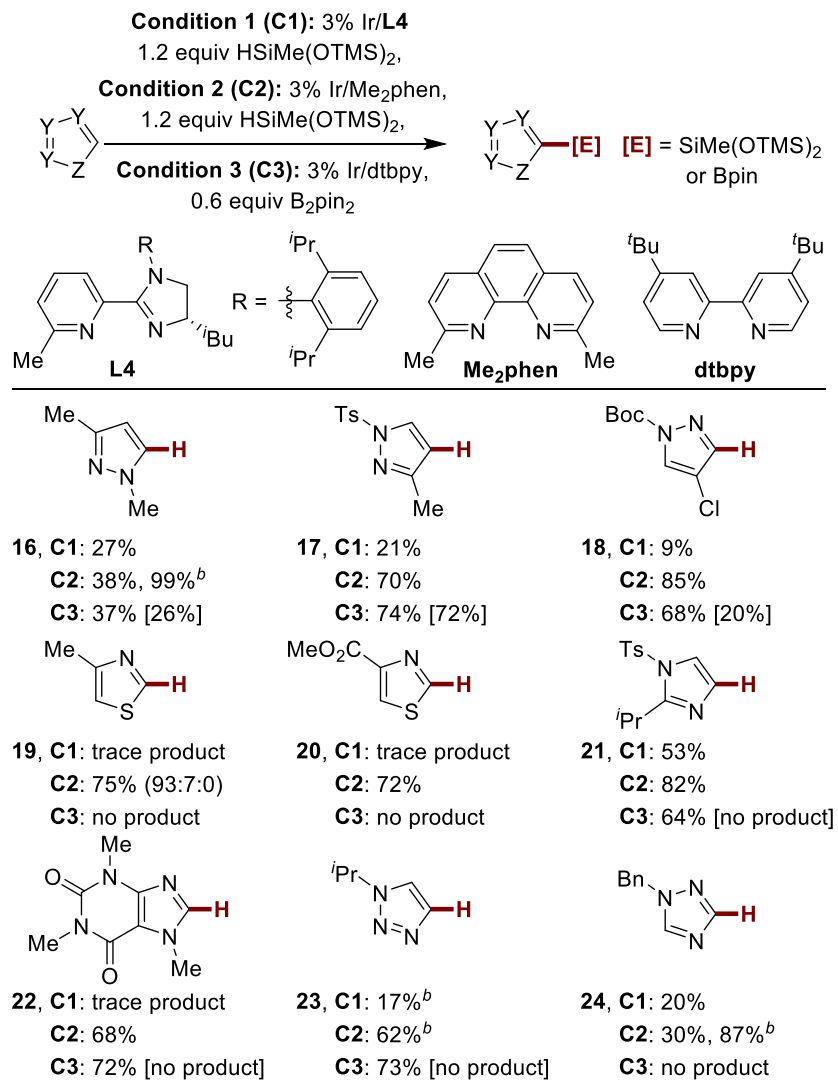

${ }^{a}$ See SI for detailed procedures. Yields correspond to ${ }^{1} \mathrm{H}$ NMR yield of desired product (desired product : undesired, mono- : undesired, di-), [yield after filtration through silica]. ${ }^{b 5} \%$ Ir/L and 2 equiv. HSiMe(OTMS) 2 .

The silylations of heteorarenes containing multiple nitrogen atoms also formed stable products in good yield. The silylation of imidazole $\mathbf{2 1}$ containing substituents at the 1 and 2 positions gave a single product from reaction at the 4 position. The imidazole unit of caffeine gave a stable product from reaction at the only available aromatic $\mathrm{C}-\mathrm{H}$ bond. Silylation of the 1,2,3- and 1,2,4-triazoles also occurred at the sterically more accessible of the two heteroaryl $\mathrm{C}-\mathrm{H}$ bonds to form stable products albeit with $5 \mathrm{~mol} \%$ catalyst.

The selectivity of the borylations of $\mathbf{1 6 , 1 8}$ and 2123 was the same as the selectivity of the silylation of these azoles, but the borylated products were unstable. The borylations of 16-18, 21, and 22 gave the functionalized product in only $37-74 \%$ yields, and reactions of 19, 20, and 24 gave no borylated product, as determined by evaluating the crude reaction 
mixtures. Moreover, the amounts of borylated products from the reactions of $\mathbf{1 6}$ and $\mathbf{1 8}$ were lower after the reaction mixtures were filtered through silica, and no borylated products were present from reactions of 21, 22 and 23 after the reaction mixtures were filtered through silica. Thus, consistent with prior literature, the products from the borylation of azoles are, in many cases, unstable to air and, in most cases, unstable to silica. 16,18 In contrast, the products from the silylation of azoles 16-23 are stable to air, moisture from solvents stored on the benchtop, and silica. The greater stability of silyl-azoles than of boryl-azoles renders the silylation of the $\mathrm{C}-\mathrm{H}$ bonds of azoles particularly useful.

\section{Scheme 1. Regio-Divergent Functionalization of Het-} eroarenes

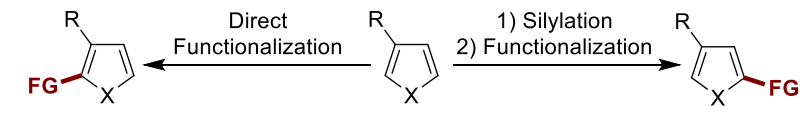

selectivity from substrate selectivity from catalyst

$\mathrm{HO}_{2} \mathrm{C} \quad$ 1) $\mathrm{LiN}\left({ }^{\prime} \mathrm{Pr}\right)_{2} \mathrm{RO}_{2} \mathrm{C} \quad$ 1) Silylation $\mathrm{EtO}_{2} \mathrm{C}$

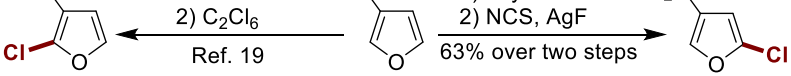

25

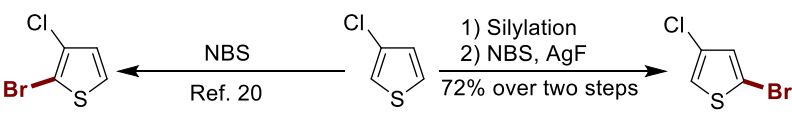

26

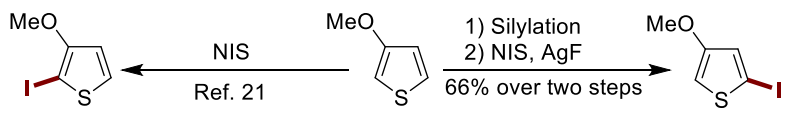

27

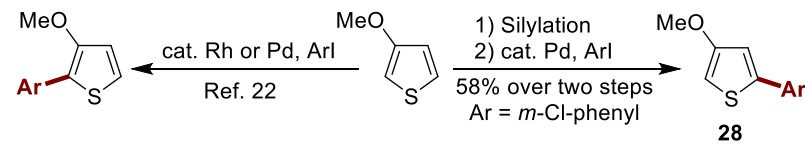

$\mathrm{EtO}_{2} \mathrm{C} \quad \mathrm{EtO}_{2} \mathrm{C} \quad$ 1) Silylation $\mathrm{EtO}_{2} \mathrm{C}$
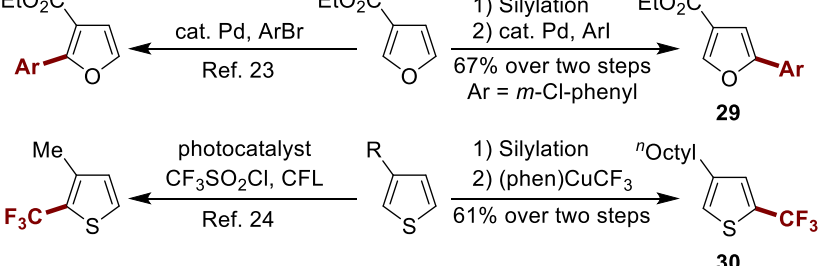

${ }^{a}$ See SI for detailed procedures.

\section{Functionalization of Heteroarylsilanes}

To demonstrate the utility of $\mathrm{C}-\mathrm{H}$ silylation to the preparation of functionalized heteroarenes, we assessed the ability to convert the silyl heteroarenes to heteroarenes that are functionalized with halogens or with aryl or trifluoromethyl groups (Scheme 1, right). In addition, we compared the products one would obtain from the combination of silylation and functionalization to those from more classical processes (Scheme 1, left). The silylation of ethyl 3-furan carboxylate, followed by functionalization with $N$-chlorosuccinimide in the presence of AgF formed ethyl 2chloro-4-furancarboxylate 25 in 63\% yield over two steps. In contrast, directed metalation of the related 3furancarboxylate, followed by quenching with hexachloroethane, is known to produce 2-chloro-3-furancarboxylate. ${ }^{19}$ The silylation of 3-chlorothiophene, followed by functionalization with $\mathrm{N}$-bromosuccinimide and AgF, formed bromide 2-bromo-4-chlorothiophene 26 in 72\% yield over two steps. In contrast, the direct bromination of 3-chlorothiophene with NBS has been reported to form 2-bromo-3-chlorothiophene. ${ }^{20}$ Likewise, the sequential silylation and iodination of 3-methoxythiophene formed 2,4-functionalized product 27 , whereas direct iodination of the unfunctionalized arene is known to form the 2,3 functionalized product. ${ }^{21}$ Heteroarylsilanes also undergo coupling reactions to form arylated products. The silylation of 3-methoxythiophene at the 5-position followed by Hiyama cross-coupling in the presence of a palladium catalyst furnished 5 -arylated product $\mathbf{2 8}$ in $58 \%$ yield over 2 steps. Previous literature reported that the direct arylation of 3-methoxythiophene, when catalyzed by rhodium or palladium complexes, leads to arylation with high selectivity for the 2-position. ${ }^{22}$ Similarly, the 5 -arylated product $\mathbf{2 9}$ formed in $67 \%$ yield over two steps by the sequential silylation and cross-coupling of ethyl 3-furancaryboxylate. In contrast, ethyl 3-furancarboxylate is known to undergo direct arylation at the 2-position in the presence of a palladium catalyst. ${ }^{23}$

Finally, the silylation of 3-methylthiophene, followed by coupling (phen) $\mathrm{CuCF}_{3}$ under an atmosphere of oxygen, formed the product from trifluoromethylation of the 5-position of the thiophene. In contrast, the direct addition of trifluoromethyl radical is known to occur at the 2-position of 3-methylthiophene. ${ }^{24}$ Due to the volatility of 2-trifluoromethyl-4-methylthiophene, this product, was characterized by the ${ }^{19} \mathrm{~F}$ NMR of the crude reaction mixture. However, to form an isolable analog, the silylation of 3-octylthiophene and coupling of the silyl thiophene with a source of trifluoromethyl group was studied, and 2-trifluoromethyl-4-octylthiophene was formed by this process in $61 \%$ yield by NMR spectroscopy and 37\% isolated yield. These results demonstrate that the silylations of heteroarenes with high sterically derived regioselectivity, followed by functionalizations of the resulting heteroarylsilanes, generate products that are distinct from those accessible from the direct functionalization of the same heteroarenes.

The silyl-azoles presented in Table 4 are more electron-deficient than the silyl-thiophenes and silylfurans in Scheme 1. To determine whether silyl-azoles are nucleophilic enough to undergo transformations that are similar to those of other silyl-arenes and heteroarenes, we conducted a series of derivatizations of silyl pyrazole 31 (Scheme 2). The reaction of $\mathbf{3 1}$ with 
NCS in the presence of AgF formed chloropyrazole 32 in $52 \%$ yield. Similarly, the reaction of $\mathbf{3 1}$ with NBS formed bromopyrazole 33 in $81 \%$ yield. Silyl-pyrazole 31 also coupled with 3,5-(MeO) $)_{2}$-phenyl triflate to form biaryl 34 in 70\% yield when catalyzed by $\mathrm{Pd}_{2} \mathrm{dba}_{3} \cdot \mathrm{CHCl}_{3}$ and BINAP. The identification of that catalyst system is discussed in greater detail below. In addition, 31 coupled with (phen) $\mathrm{CuCF}_{3}$ under an atmosphere of oxygen to form 1-Ts-3-Me-4- $\mathrm{CF}_{3}$ pyrazole (35) in 50\% yield. These results demonstrate that the silylation of azoles, followed by the functionalization of the product, also can lead to products containing a diverse array of functional groups.

\section{Scheme 2. Transformations of Silyl-Pyrazole ${ }^{a}$}
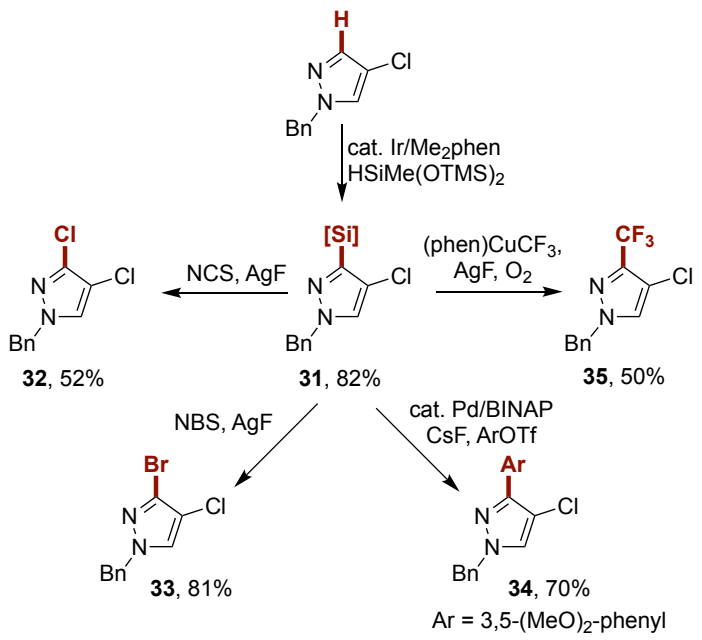

${ }^{a}$ See SI for detailed procedures.

Suzuki couplings are widely used, in part, because the aryl boronic acid and ester reagents are stable to air and moisture and can be stored. However, as discussed, the boronic acids or esters of azoles are often unstable. Therefore, coupling of the more stable silyl derivatives of five-membered heteroarenes would be valuable. We found that the silyl-thiophenes and silylfurans that result from the silylation of $\mathrm{C}-\mathrm{H}$ bonds couple with aryl iodides in the presence of $\mathrm{AgF}$ and catalytic amounts of $\mathrm{Pd}\left(\mathrm{P}^{t} \mathrm{Bu}_{3}\right)_{2}$. However, under similar conditions, only $10 \%$ of the product from the reaction of the silyl thiazole 2-silyl-4-thiazolecarboxylate methyl ester formed from coupling the representative partner $m$-Cl-iodobenzene (Table 5). After conducting reactions with a series of fluoride additives and phosphine ligands, we found that a high yield of biaryl product (85\%) was obtained from reactions with $\mathrm{CsF}$ as activator and the combination of $\mathrm{Pd}_{2} \mathrm{dba}_{3} \cdot \mathrm{CHCl}_{3}$ and BINAP as catalyst. The 2-silyl-4thiazolecarboxylate methyl ester also coupled with heteroaryl bromides catalyzed by the same system to form biaryls $\mathbf{3 7}$ and $\mathbf{3 8}$ in $76 \%$ and $50 \%$ yield. Although bithiazole 39 formed in a modest $22 \%$ yield from the same coupling of the silyl thiazole with 2bromothiazole, this reaction has not been accomplished previously by Suzuki coupling of the analogous boronate, presumably due to the instability of this boronate. As noted in the previous paragraph, these conditions were also suitable for the coupling of the silyl-pyrazole 31 to form 3-aryl pyrazole 34. Thus, the combination of CsF activator and BINAP-ligated palladium is an effective system to catalyze the coupling of silylazoles with aryl halides under mild conditions.

Table 5. Hiyama Coupling of Silyl-Azoles ${ }^{a}$

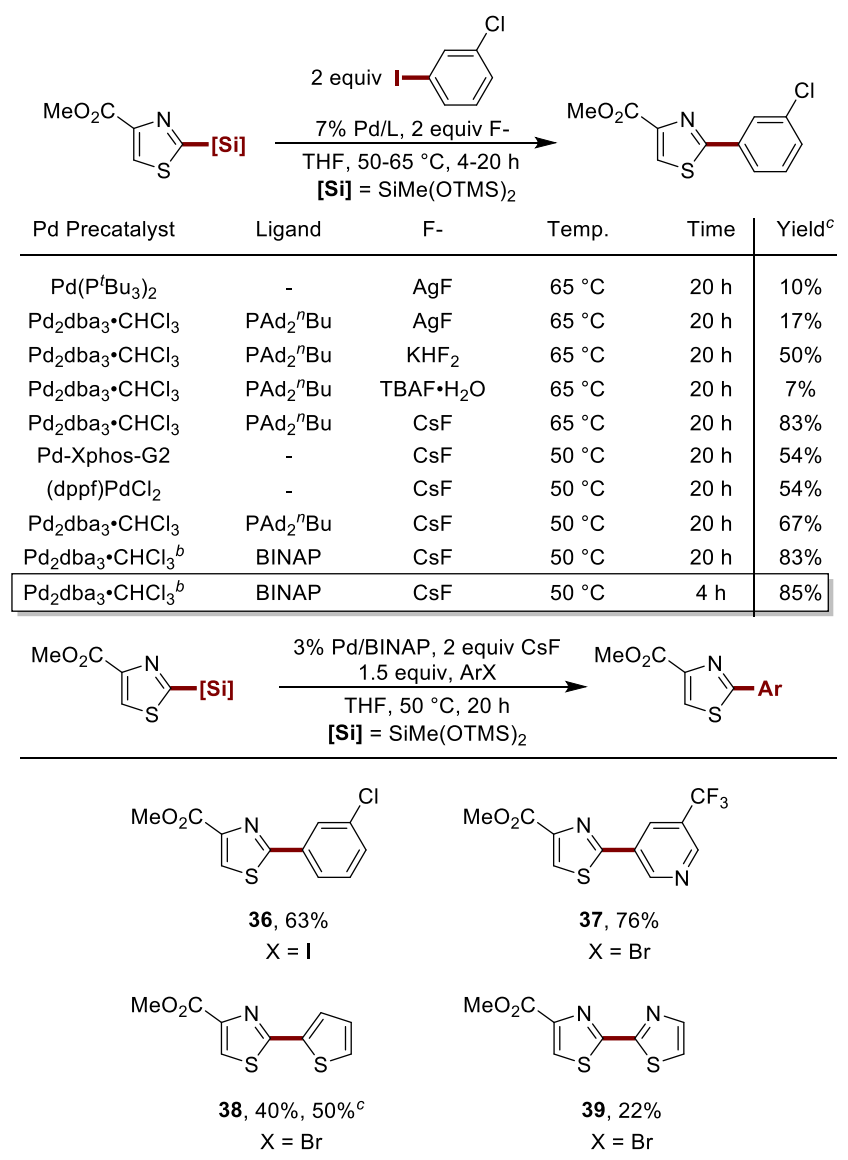

${ }^{a}$ See SI for detailed procedures. ${ }^{b} 3 \% \mathrm{Pd} / \mathrm{L}{ }^{c}$ Determined by ${ }^{1} \mathrm{H}$ NMR spectroscopy with dibromomethane internal standard

\section{Origin of Regioselectivity}

To determine the elementary steps controlling the high selectivity for the silylation of unhindered $\mathrm{C}-\mathrm{H}$ bonds of heteroarenes catalyzed by complexes of $\mathbf{L 4}$, we conducted studies on the reactions of deuterated silane with 3-chlorothiophene (7). The reactions of an excess of $\mathbf{7}$ with deuterated silane catalyzed by iridium and $\mathrm{Me}_{2}$ phen or $\mathbf{L} \mathbf{4}$ exchanged deuterium between the silane Si-D bond and the 2-positions and 5positions of the heteroarene to form deuterated products $\mathbf{7} \mathbf{c}$ and $\mathbf{7 d}$, respectively. This reaction occurred in concert with the formation of silylated products $7 \mathbf{a}$ and $\mathbf{7 b}$. After $15 \mathrm{~min}$ at $65^{\circ} \mathrm{C}$, the amounts of deuterated products $\mathbf{7 c}$ and $\mathbf{7 d}$ were greater than the amounts of silylated products $\mathbf{7 a}$ and $\mathbf{7 b}$ by factors of 
about 2:1 and 6:1 for the catalysts containing ligands $\mathrm{Me}_{2}$ phen and L4, respectively. This incorporation of deuterium into the heteroarene reactant indicates that cleavage of the $\mathrm{C}-\mathrm{H}$ bond is reversible.

Table 5. Reactions of Deuterated Silane with Excess Arene $^{a}$

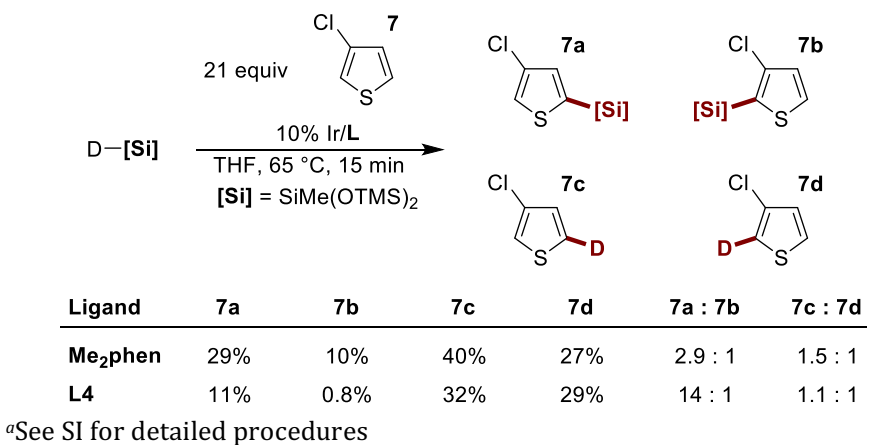

The ratio of deuterated products was distinct from the ratio of silylated products. The reaction catalyzed by the complex of $\mathrm{Me}_{2}$ phen formed deuterated products $\mathbf{7 c}$ and $\mathbf{7 d}$ in a ratio of $1.5: 1$ but formed the silylated products $\mathbf{7 a}$ and $\mathbf{7 b}$ in a higher ratio of $2.9: 1$ (Table 5). The reaction catalyzed by a complex of $\mathbf{L 4}$ formed the deuterated products $\mathbf{7} \mathbf{c}$ and $\mathbf{7 d}$ in a ratio of only 1.1:1 but formed the silylated products $\mathbf{7} \mathbf{a}$ and $\mathbf{7 b}$ in a high ratio of 14:1. Thus, the cleavage of $\mathrm{C}-\mathrm{H}$ bonds by the catalyst that contains $\mathbf{L} 4$ is less regioselective than that of the catalyst that contains $\mathrm{Me}_{2}$ phen, but the formation of silylated products by the catalyst that contains $\mathbf{L 4}$ occurs with much greater regioselectivity than that of the catalyst that contains $\mathrm{Me}_{2}$ phen. These results demonstrate that the regioselectivity of these two catalysts for the silylation of $\mathrm{C}-\mathrm{H}$ bonds does not reflect the propensity of the catalysts to cleave $\mathrm{C}-\mathrm{H}$ bonds. Rather, the selectivity of the silylation reaction is controlled by the relative rates of formation of the carbon-silicon bond from 2- or 5-heteroaryliridium intermediates that are formed reversibly. These experimental results resemble the computations reported by Sakaki for the selectivity of the borylations of $\beta$ over $\alpha \mathrm{C}-\mathrm{H}$ bonds in THF, as well as the experiments and computations our group reported previously on the origins of selectivity of $\mathrm{Rh}$ catalyzed borylations and silylations of primary over secondary alkyl $\mathrm{C}-\mathrm{H}$ bonds. ${ }^{25}$

\section{Conclusion}

In summary, we have developed a method for the silylation of heteroaromatic compounds with high sterically derived regioselectivity. In many cases, these high selectivities are achieved with a new pyridyl-imidazoline ligand $\mathbf{L 4}$. The selectivity of this silylation to form functionalized products from reaction at the least sterically hindered position of five-membered heteroarenes is much higher than that of previously reported borylations of these heteroarenes, and the products formed are much more stable than the analogous boronates. Moreover, functionalizations of the heteroarylsilanes form products with substitution patterns that are orthogonal to those that have been reported by traditional methods and other catalytic processes. Mechanistic studies showed that the rates of formation of the carbon-silicon bond from isomeric heteoraryliridium complexes influences the selectivities of the silylation reactions more than the rates of the cleavage of carbon-hydrogen bonds to form these complexes and that the selectivities from the reactions catalyzed by the combination of iridium and the pyridyl-imidazoline ligand $\mathbf{L 4}$ result almost exclusively from the rates of formation of the carbon-silicon bond. Additional studies on the mechanism and origin of high selectivity of the silylation reaction with catalysts that contain imidazoline ligands are ongoing in our laboratory.

\section{ASSOCIATED CONTENT}

\section{Supporting Information}

Experimental procedures and spectroscopic data on the reaction products. The Supporting Information is available free of charge on the ACS Publications website.

\section{AUTHOR INFORMATION}

\section{Corresponding Author}

*jhartwig@berkeley.edu

\section{Author Contributions}

‡These authors contributed equally.

\section{Notes}

The authors declare no competing financial interests.

\section{ACKNOWLEDGMENT}

We gratefully acknowledge financial support from the NIH (R35GM130387). We thank the College of Chemistry's NMR facility for resources provided and the staff for their assistance. Instruments in CoC-NMR are supported in part by the NIH (S100D024998). C.K., C.Z.R. and E.V.K. thank Zhewei Chen, Dr. Ala Bunescu and Dr. Raphael Oeschger for helpful discussions.

\section{REFERENCES}

1. (a) Godula, K.; Sames, D. C-H Bond Functionalization in Complex Organic Synthesis. Science 2006, 312, 67. (b) Davies, H. M. L.; Du Bois, J.; Yu, J. Q. C-H Functionalization in organic synthesis. Chem. Soc. Rev. 2011, 40, 1855. (c) Gutekunst, W. R.; Baran, P. S. C-H functionalization logic in total synthesis. Chem. Soc. Rev. 2011, 40, 1976. (d) Yamaguchi, J.; Yamaguchi, A. D.; Itami, K. C-H Bond Functionalization: Emerging Synthetic Tools for Natural Products and Pharmaceuticals. Angew. Chem. Int. Ed. 2012, 51, 8960. (e) Wencel-Delord, J.; Glorius, F. C-H bond activation enables the rapid construction and late-stage diversification of functional molecules. Nat. Chem. 2013, 5, 369. (f) Hartwig, J. F. Evolution of C-H Bond Functionalization from Methane to Methodology. J. Am. Chem. Soc. 2016, 138, 2. (g) McMurray, L.; O'Hara, F.; Gaunt, M. J. Recent developments in natural product synthesis using metal-catalysed C-H bond functionalisation. Chem. Soc. Rev. 2011, 40, 1885. (h) White, M. C. Adding Aliphatic C-H Bond Oxidations to Synthesis. Science 2012, 335, 807. 
2. (a) Neufeldt, S. R.; Sanford, M. S. Controlling Site Selectivity in Palladium-Catalyzed C-H Bond Functionalization. Acc. Chem. Res. 2012 45, 936. (b) Hartwig, J. F. Catalyst-Controlled Site-Selective Bond Activation. Acc. Chem. Res. 2017, 50, 549.

3. (a) Lyons, T. W.; Sanford, M. S. Palladium-Catalyzed Ligand-Directed C-H Functionalization Reactions. Chem. Rev. 2010, 110, 1147 (b) Ros, A.; Fernandez, R.; Lassaletta, J. M. Functional group directed CH borylation. Chem. Soc. Rev. 2014, 43, 3229. (c) Engle, K. M.; Mei, T.S.; Wasa, M.; Yu, J.-Q. Weak Coordination as a Powerful Means for Developing Broadly Useful C-H Functionalization Reactions. Acc. Chem. Res. 2012, 45, 788. (d) Wang, P.; Farmer, M. E.; Huo, X.; Jain, P.; Shen, P.-X.; Ishoey, M.; Bradner, J. E.; Wisniewski, S. R.; Eastgate, M. D.; Yu, J.-Q. Ligand-Promoted Meta-C-H Arylation of Anilines, Phenols, and Heterocycles. J. Am. Chem. Soc. 2016, 138, 9269. (e) Wan, L.; Dastbaravardeh, N.; Li, G.; Yu, J.-Q. Cross-Coupling of Remote meta-C-H Bonds Directed by a U-Shaped Template. J. Am. Chem. Soc. 2013, 135, 18056. (f) Dai, H.-X.; Li, G.; Zhang, X.-G.; Stepan, A. F.; Yu, J.-Q. Pd(II)-Catalyzed ortho- or meta-C-H Olefination of Phenol Derivatives. J. Am. Chem. Soc. 2013, 135, 7567. (g) Leow, D.; Li, G.; Mei, T. S.; Yu, J. Q. Activation of remote meta-C-H bonds assisted by an end-on template. Nature $\mathbf{2 0 1 2}$ 486, 518. (h) Li, M.; Shang, M.; Xu, H.; Wang, X.; Dai, H.-X.; Yu, J.-Q. Remote Para-C-H Acetoxylation of Electron-Deficient Arenes. Org. Lett. 2019, 21, 540. (i) Wang, X.; Leow, D.; Yu, J.-Q. Pd(II)-Catalyzed paraSelective C-H Arylation of Monosubstituted Arenes. J. Am. Chem. Soc. 2011, 133, 13864.

4. (a) Wedi, P.; van Gemmeren, M. Arene-Limited Nondirected C-H Activation of Arenes. Angew. Chem. Int. Ed. 2018, 57, 13016. (b) Hartwig, J. F.; Larsen, M. A. Undirected, Homogeneous C-H Bond Functionalization: Challenges and Opportunities. ACS Cent. Sci. 2016, 2, 281.

5. (a) Olah, G. A. Aromatic substitution. XXVIII. Mechanism of electrophilic aromatic substitutions. Acc. Chem. Res. 1971, 4, 240. (b) Boursalian, G. B.; Ham, W. S.; Mazzotti, A. R.; Ritter, T. Charge-transfer-directed radical substitution enables para-selective $\mathrm{C}-\mathrm{H}$ functionalization. Nat Chem 2016, 8, 810.

6. (a) Hartwig, J. F. Borylation and Silylation of C-H Bonds: A Platform for Diverse C-H Bond Functionalizations. Acc. Chem. Res. 2012 , 45, 864. (b) Mkhalid, I. A. I.; Barnard, J. H.; Marder, T. B.; Murphy, J. M.; Hartwig, J. F. C-H Activation for the Construction of C-B Bonds. Chem. Rev. 2010, 110, 890. (c) Cheng, C.; Hartwig, J. F. Catalytic Silylation of Unactivated C-H Bonds. Chem. Rev. 2015, 115, 8946. (d) Hartwig, J. F. Regioselectivity of the borylation of alkanes and arenes. Chem. Soc. Rev. 2011, 40, 1992.

7. (a) Chotana, G. A.; Kallepalli, V. A.; Maleczka, R. E. J.; Smith, M. R. I. Iridium-Catalyzed Borylation of Thiophenes: Versatile, Synthetic Elaboration Founded on Selective C-H Funtionalization. Tetrahedron 2008, 64, p 6103. (b) Cheng, C.; Hartwig, J. F. Iridium-Catalyzed Silylation of Aryl C-H Bonds. J. Am. Chem. Soc. 2015, 137, 592.

8. Miller, S. L.; Chotana, G. A.; Fritz, J. A.; Chattopadhyay, B.; Maleczka, R. E.; Smith, M. R. C-H Borylation Catalysts that Distinguish Between Similarly Sized Substituents Like Fluorine and Hydrogen. Org. Lett. 2019, 21, 6388.

9. Welsch, M. E.; Snyder, S. A.; Stockwell, B. R. Privileged scaffolds for library design and drug discovery. Curr. Opin. Chem. Biol. 2010, 14 347.

10. (a) Rayment, E. J.; Mekareeya, A.; Summerhill, N.; Anderson, E. A. Mechanistic Study of Arylsilane Oxidation through 19F NMR Spectroscopy. J. Am. Chem. Soc. 2017, 139, 6138. (b) Rayment, E. J.; Summerhill, N.; Anderson, E. A. Synthesis of Phenols via Fluoride-free Oxidation of Arylsilanes and Arylmethoxysilanes. J. Org. Chem. 2012, 77, 7052. (c) Bracegirdle, S.; Anderson, E. A. Arylsilane oxidation-new routes to hydroxylated aromatics. Chem. Commun. 2010, 46, 3454. (d) Maleczka, R. E.; Shi, F.; Holmes, D.; Smith, M. R. I. C-H activation/borylation/oxidation: A one-pot unified route to meta-substituted phenols bearing ortho-/para-directing groups. J. Am. Chem. Soc. 2003, 125, 7792.

11. Tzschucke, C. C.; Murphy, J. M.; Hartwig, J. F. Arenes to anilines and aryl ethers by sequential iridium-catalyzed borylation and coppercatalyzed coupling. Org. Lett. 2007, 9, 761.

12. (a) Qiao, J. X.; Lam, P. Y. S. Copper-promoted carbon-heteroatom bond cross-coupling with boronic acids and derivatives. Synthesis, 2011, 829. (b) Morstein, J.; Kalkman, E. D.; Bold, C.; Cheng, C.; Hartwig, J. F. Copper-Mediated C-N Coupling of Arylsilanes with Nitrogen Nucleophiles. Org. Lett. 2016, 18, 5244.

13. Liskey, C. W.; Liao, X.; Hartwig, J. F. Cyanation of Arenes via Iridium-Catalyzed Borylation. J. Am. Chem. Soc. 2010, 132, 11389.
14. (a) Partridge, B. M.; Hartwig, J. F. Sterically Controlled Iodination of Arenes via Iridium-Catalyzed C-H Borylation. Org. Lett. 2013, 15, 140. (b) Murphy, J. M.; Liao, X.; Hartwig, J. F. Meta Halogenation of 1,3Disubstituted Arenes via Iridium-Catalyzed Arene Borylation. J. Am. Chem. Soc. 2007, 129, 15434.

15. (a) Robbins, D. W.; Hartwig, J. F. A C-H Borylation Approach to Suzuki-Miyaura Coupling of Typically Unstable 2-Heteroaryl and Polyfluorophenyl Boronates. Org. Lett. 2012, 14, 4266. (b) Miyaura, N., Metal-Catalyzed Cross-Coupling Reactions of Organoboron Compounds with Organic Halides. In Metal-Catalyzed Cross-Coupling Reactions, Wiley-VCH Verlag GmbH: 2008; pp 41. (c) Brown, D. G.; Boström, J. Analysis of Past and Present Synthetic Methodologies on Medicinal Chemistry: Where Have All the New Reactions Gone? J. Med. Chem. 2016, 59, 4443. (d) Nakao, Y.; Hiyama, T. Silicon-based cross-coupling reaction: an environmentally benign version. Chem. Soc. Rev. 2011, 40 , 4893. (e) Denmark, S. E.; Ambrosi, A. Why You Really Should Consider Using Palladium-Catalyzed Cross-Coupling of Silanols and Silanolates. Org. Process Res. Dev. 2015, 19, 982.

16. (a) Cox, P. A.; Leach, A. G.; Campbell, A. D.; Lloyd-Jones, G. C. Protodeboronation of Heteroaromatic, Vinyl, and Cyclopropyl Boronic Acids: pH-Rate Profiles, Autocatalysis, and Disproportionation. J. Am. Chem. Soc. 2016, 138, 9145. (b) Denmark, S. E.; Regen, C. S. PalladiumCatalyzed Cross-Coupling Reactions of Organosilanols and Their Salts: Practical Alternatives to Boron- and Tin-Based Methods. Acc. Chem. Res. 2008, 41, 1486. (c) Lennox, A. J.; Lloyd-Jones, G. C. Selection of boron reagents for Suzuki-Miyaura coupling. Chem. Soc. Rev. 2014, 43, 412.

17. (a) Ishiyama, T.; Takagi, J.; Yonekawa, Y.; Hartwig, J. F.; Miyaura, $\mathrm{N}$. Iridium-catalyzed direct borylation of five-membered heteroarenes by bis(pinacolato)diboron: Regioselective, stoichiometric, and room temperature reactions. Adv. Synth. Catal. 2003, 345, 1103. (b) Ishiyama, T.; Takagi, J.; Ishida, K.; Miyaura, N.; Anastasi, N.; Hartwig, J. F. Mild iridium-catalyzed borylation of arenes. High turnover numbers, room temperature reactions, and isolation of a potential intermediate.J. Am. Chem. Soc. 2002, 124, 390. (c) Karmel, C.; Chen, Z.; Hartwig, J. F. Iridium-Catalyzed Silylation of C-H Bonds in Unactivated Arenes: A Sterically Encumbered Phenanthroline Ligand Accelerates Catalysis. J. Am. Chem. Soc. 2019, 141, 7063.

18. Larsen, M. A.; Hartwig, J. F. Iridium-Catalyzed C-H Borylation of Heteroarenes: Scope, Regioselectivity, Application to Late-Stage Functionalization, and Mechanism. J. Am. Chem. Soc. 2014, 136, 4287.

19. Schnute, M. E.; Brideau, R. J.; Collier, S. A.; Cudahy, M. M.; Hopkins, T. A.; Knechtel, M. L.; Oien, N. L.; Sackett, R. S.; Scott, A.; Stephan, M. L. Synthesis of 4-oxo-4, 7-dihydrofuro [2, 3-b] pyridine-5-carboxamides with broad-spectrum human herpesvirus polymerase inhibition. Bioorg. Med. Chem. Lett. 2008, 18, 3856.

20. Mader, M. M.; Shih, C.; Considine, E.; De Dios, A.; Grossman, C. S.; Hipskind, P. A.; Lin, H.-S.; Lobb, K. L.; Lopez, B.; Lopez, J. E. Acyl sulfonamide anti-proliferatives. Part 2: Activity of heterocyclic sulfonamide derivatives. Bioorg. Med. Chem. Lett. 2005, 15, 617.

21. Grolleau, J.; Frère, P.; Gohier, F. Clean and Efficient Iodination of Thiophene Derivatives. Synthesis 2015, 47, 3901.

22. (a) Yanagisawa, S.; Sudo, T.; Noyori, R.; Itami, K. Direct C-H arylation of (hetero)arenes with aryl iodides via rhodium catalysis. J. Am. Chem. Soc. 2006, 128, 11748. (b) Borghese, A.; Geldhof, G.; Antoine, L. Direct C-H arylation of 3-methoxythiophene catalyzed by Pd. Application to a more efficient synthesis of $\pi$-alkoxy-oligothiophene derivatives. Tetrahedron Lett. 2006, 47, 9249.

23. Glover, B.; Harvey, K. A.; Liu, B.; Sharp, M. J.; Tymoschenko, M. F. Regioselective Palladium-Catalyzed Arylation of 3-Carboalkoxy Furan and Thiophene. Org. Lett. 2003, 5, 301.

24. Nagib, D. A.; MacMillan, D. W. C. Trifluoromethylation of arenes and heteroarenes by means of photoredox catalysis. Nature 2011, 480, 224.

25. (a) Zhong, R.-L.; Sakaki, S. sp3 C-H Borylation Catalyzed by Iridium(III) Triboryl Complex: Comprehensive Theoretical Study of Reactivity, Regioselectivity, and Prediction of Excellent Ligand. J. Am. Chem. Soc. 2019, 141, 9854. (b) Wei, C. S.; Jiménez-Hoyos, C. A.; Videa, M. F.; Hartwig, J. F.; Hall, M. B. Origins of the Selectivity for Borylation of Primary over Secondary C-H Bonds Catalyzed by Cp*-Rhodium Complexes. J. Am. Chem. Soc. 2010, 132, 3078. (c) Karmel, C.; Li, B.; Hartwig, J. F. Rhodium-Catalyzed Regioselective Silylation of Alkyl C-H Bonds for the Synthesis of 1,4-Diols. J. Am. Chem. Soc. 2018, 140, 1460. 
Insert Table of Contents artwork here

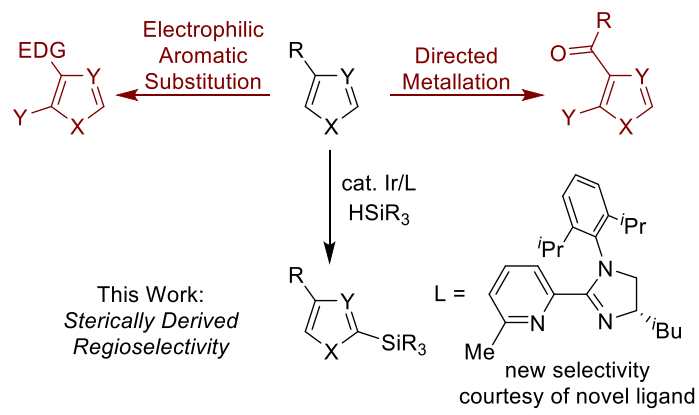

